\title{
RECENT PROGRESS IN PSYCHIATRY
}

It is hoped to issue a special number of the Journal of Mental Science for July, 1943, dealing with " Recent Progress in Psychiatry."

\section{CONTENTS}

CHAP.

I. INTRODUCTION.

G. W. T. H. Fleming, M.R.C.S., L.R.C.P., D.P.M.

2. Mental Health Services.

A. A. W. Petrie, M.D., B.S., F.R.C.P., F.R.C.S.Ed., D.P.M.

3. Genetics.

E. T. O. Slater, M.A., M.D.Camb., M.R.C.P.

4. Anatomy of the Nervous System. W. E. le Gros Clark, F.R.S., D.Sc.Lond., F.R.C.S.

5. Physiology of the Nervous System. F. L. Golla, M.A., M.B.Oxon., F.R.C.P.

6. ELECTROENCEPHALUGRAPHY. W. Grey Walter, M.A.Cantab.

7. BIochemistry of the Nervous - System. Derek Richter, D.Sc.

8. Vitamin Deficiencies in Psychiatry AND THEIR TREATMENT.

G. W. T. H. Fleming, M.R.C.S., L.R.C.P., D.P.M., and A. Meyer, M.D.Bonn.

9. ENDOCRINOLOGY.

M. Reiss, M.D., D.Sc.Prague.

io. Mental Testing.

M. B. Brody, M.D.Sheff., D.P.M.

11. Some Aspects of Perceptual and CONCEPTUAL ThOUght.

E. L. Hutton, M.B., B.S., D.P.M.

12. PSYChOPATHOLOGY.

S. M. Coleman, it.R.C.S., L.R.C.P., D.P.M.

13. NEUROPATHOLOGY.

A. Meyer, M.D.Bonn.

14. SCHIzOPHRENIA.

W. M a y e r-Gros s, M . D .Heidelberg, L.R.C.P., L.R.C.S.Ed., and J. P. Moore, M.Dubl, M.R.C.P.I.

15. Depressive States. Aubrey J. Lewis, M.D.Adelaide, F.R.C.P.

16. Manic States.

Desmond Curran, M.B.Camb., F.R.C.P., D.P.M.
СНАP.

17. Psychopathic Personality.

Desmond Curran, M.B.Camb., F.R.C.P., D.P.M., and W. P. Mallinson, M.A., B.M.Oxon., M.R.C.P

18. The Psychoneuroses.

W. Hewitt Gillespie, M.D.Ed., Dipl. Psych., M.R.C.P.

I9. PSYCHOTHERAPY.

H. Crichton-Miller, M.A., M.D.Ed., F.R.C.P.

20. Senile and Pre-senile Psychoses. W. Mayer-Gross, M.D.Heidelberg.

2I. EPILEPSY

F. L. Golla, M.A., M.B.Oxon., F.R.C.P.

22. Organic Psychoses.

E. Guttmann, M.D.Munich, L.R.C.P., L.R.C.S.Ed.

23. HEAD INJURIES.

E. Guttmann, M.D.Munich, L.R.C.P. L.R.C.S.Ed.

24. NEUROSYPHILIS AND ITS TREATMENT.

W. D. Nicol, M.B., F.R.C.P.Lond., D.P.M., and E. L. Hutton, M.B., B.S., D.P.M.

25. Child Psychiatry.

E. Mildred Creak, M.D.Lond., M.R.C.P. D.P.M.

26. Crime and Delinguency.

W. Norwood East, M.D.Lond., F.R.C.P.

27. Mental Deficiency.

- L.S. Penrose, M.A., M.D.Camb., M.R.C.S., L.R.C.P.

28. Continuous Narcosis.

R. Ström-Olsen, M.D.Wales, M.R.C.S., D.P.M.

29. Endocrine Treatment in Psychiatry. R. E. Hemphill, M.B.Dubl., D.P.M.

3o. Convulsion Treatment

L. C. Cook, M.D., M.R.C.S., L.R.C.P., D.P.M.

31. InSUlin TREatMent.

T. Tennent, M.D.Glas., D.P.H., D.P.M.

32. Pre-frontal Leucotomy.

G. W. T. H. Fleming, M.R.C.S., L.R.C.P. D.P.M.

33. Legal Aspects of Psychiatry.

P. K. McCowan, M.D.Ed., F.R.C.P., D.P.M. 